(2) OPEN ACCESS

\title{
Digestive system is a potential route of COVID-19: an analysis of single-cell coexpression pattern of key proteins in viral entry process
}

\author{
Hao Zhang, ${ }^{1,2,3}$ Zijian Kang, ${ }^{1,3}$ Haiyi Gong, ${ }^{2,3}$ Da Xu, ${ }^{3,4}$ Jing Wang, ${ }^{5}$ Zhixiu Li, ${ }^{6}$ Zifu Li, ${ }^{5}$ \\ Xinggang Cui, ${ }^{4}$ Jianru Xiao, ${ }^{2}$ Jian Zhan, ${ }^{7}$ Tong Meng (1) , 3,8,9 Wang Zhou, ${ }^{3,10}$ \\ Jianmin Liu, ${ }^{5}$ Huji Xu $u^{1,10,11}$
}

Additional material is published online only. To view please visit the journal online (http://dx.doi.org/10.1136/ gutjnl-2020-320953).

For numbered affiliations see end of article.

\section{Correspondence to}

Professor Huji Xu, Changzheng Hospital, Second Military Medical University, Shanghai 200003, China;

xuhuji@smmu.edu.cn, Professor Jianmin Liu, Changhai Hospital, Second Military Medical University, Shanghai, China; chstroke@163.com, DrWang Zhou, Peking-Tsinghua Center for Life Sciences, Tsinghua University, Beijing, China; brilliant212@163.com, Dr Tong Meng, Tongji Hospital affiliated to Tongji University School of Medicine, Shanghai, China; mengtong@medmail.com.cn and Dr Jian Zhan, Institute for Glycomics, Griffith University, Southport, QLD, Australia; j.zhan@griffith.edu.au

$\mathrm{HZ}, \mathrm{ZK}, \mathrm{HG}$ and DX contributed equally.

$J Z$, TM, WZ, JL and HX

contributed equally.

$H Z, Z K, H G$ and $D X$ are joint first authors.

$J Z, T M, W Z$, JL and $H X$ are joint senior authors.

Received 21 February 2020 Revised 24 March 2020

Accepted 25 March 2020

Published Online First

2 April 2020

\section{Check for updates}

(C) Author(s) (or their employer(s)) 2020. Re-use permitted under CC BY-NC. No commercial re-use. See rights and permissions. Published by BMJ.

To cite: Zhang $\mathrm{H}$, Kang $\mathrm{Z}$, Gong $\mathrm{H}$, et al. Gut

2020;69:1010-1018.

\section{ABSTRACT}

Objective Since December 2019, a newly identified coronavirus (severe acute respiratory syndrome coronavirus (SARS-CoV-2)) has caused outbreaks of pneumonia in Wuhan, China. SARS-CoV-2 enters host cells via cell receptor ACE II (ACE2) and the transmembrane serine protease 2 (TMPRSS2). In order to identify possible prime target cells of SARS-CoV-2 by comprehensive dissection of ACE2 and TMPRSS2 coexpression pattern in different cell types, five datasets with single-cell transcriptomes of lung, oesophagus, gastric mucosa, ileum and colon were analysed. Design Five datasets were searched, separately integrated and analysed. Violin plot was used to show the distribution of differentially expressed genes for different clusters. The ACE2-expressing and TMPRRSS2expressing cells were highlighted and dissected to characterise the composition and proportion.

Results Cell types in each dataset were identified by known markers. ACE2 and TMPRSS2 were not only coexpressed in lung AT2 cells and oesophageal upper epithelial and gland cells but also highly expressed in absorptive enterocytes from the ileum and colon. Additionally, among all the coexpressing cells in the normal digestive system and lung, the expression of ACE2 was relatively highly expressed in the ileum and colon.

Conclusion This study provides the evidence of the potential route of SARS-CoV-2 in the digestive system along with the respiratory tract based on singlecell transcriptomic analysis. This finding may have a significant impact on health policy setting regarding the prevention of SARS-CoV-2 infection. Our study also demonstrates a novel method to identify the prime cell types of a virus by the coexpression pattern analysis of single-cell sequencing data.

\section{INTRODUCTION}

At the end of 2019, a rising number of patients with novel coronavirus pneumonia (coronavirus disease (COVID-19)) with unknown pathogenesis emerged in one of the largest cities of China, Wuhan, and quickly spread throughout the whole country. ${ }^{1} \mathrm{~A}$ novel coronavirus was then isolated from human airway epithelial cells and was named severe acute respiratory syndrome coronavirus (SARS-CoV-2). ${ }^{2}$

\section{Significance of this study}

What is already known on this subject?

- Both of ACE2 and transmembrane serine protease 2 (TMPRSS2) are key proteins of severe acute respiratory syndrome coronavirus (SARS-CoV-2) cell entry process. Coexpression of these two proteins in the same cell is critical for viral entry.

- Currently, the prime target cells of SARS-CoV-2 are unclear due to incomplete knowledge of the ACE2 and TMPRSS2 coexpression pattern in the cells of respiratory tract and digestive tract.

- Though droplet transmission is considered as the main route of transmission, the other transmission routes remain unclear.

What are the new findings?

- Alveolar type 2 cells are the main cell type coexpressing ACE2 and TMPRSS2 in lung tissue.

- In addition, ACE2 and TMPRSS2 are also coexpressed in both upper epithelial and gland cells from oesophagus and absorptive enterocytes from ileum and colon.

How might it impact on clinical practice in the foreseeable future?

- This study provides the evidence of a potential route of SARS-CoV-2 in the digestive system along with the respiratory tract based on single-cell transcriptomic analysis.

- Faecal-oral transmission is a possible route of SARS-CoV-2 transmission. As such, these data may have significant impact for healthy policy setting regarding the prevention of SARS-CoV-2 infection.

The complete genome sequences have revealed that SARS-CoV-2 shares $86.9 \%$ nucleotide sequence identity with a SARS-like coronavirus detected in bats (bat-SL-CoVZC45, MG772933.1). This study suggested that SARS-CoV-2 is a specie of SARSrelated coronaviruses (SARSr-CoV) by pairwise protein sequence analysis. ${ }^{23}$

Regarding the clinical manifestations of SARS-CoV- 2 infection, fever and cough are the most common symptoms at onset. ${ }^{34}$ In addition, $2 \%-10 \%$ 
of patients with COVID-19 had gastrointestinal symptoms such as vomiting, diarrhoea and abdominal pain. ${ }^{56}$ However, little is known about why and how SARS-CoV-2 induces enteric symptoms. In addition, it is currently unknown whether SARS-CoV-2 can be transmitted through the digestive tract in addition to through the respiratory tract. ${ }^{3}$

The prerequisite of coronavirus infection is its entrance into a host cell. During this process, the spike (S) glycoprotein recognises host cell receptors and induces the fusion of viral and cellular membranes. ${ }^{7}$ In SARS-CoV-2 infection, a metallopeptidase, ACE II (ACE2) has been proven to be the cell receptor, similar to SARS-CoV infection. ${ }^{8-11}$ Currently, evidence shows that SARS-CoV-2 requires ACE2 to enter the host cell. ${ }^{9}$ Beside, the transmembrane serine protease (TMPRSS2) is the main host cell protease which cleave the $S$ protein of human coronaviruses on the cell membrane, allowing the virus to release fusion peptide for membrane fusion. ${ }^{12}$ Therefore, coexpression of ACE2 and TMPRSS2 is critical for the cell entry process of SARS-CoV-2.

To explore the susceptible cell types and potential infection routes of SARS-CoV-2, we analysed the coexpression pattern of ACE2 and TMPRSS2 in different cell types in both normal human lungs and the gastrointestinal system by single-cell transcriptomics based on public databases. A striking finding is that ACE2 and TMPRSS2 are coexpressed not only in lung alveolar type 2 (AT2) cells, but also in oesophagus upper cells upper epithelial and gland cells and absorptive enterocytes from the ileum and colon. These findings suggest that the enteric symptoms of COVID-19 may be associated with the invasion of SARS-CoV-2 into the ACE2 and TMPRSS2 coexpressing enterocytes. Our single-cell transcriptomic study for the first time provides the evidence which indicates that the digestive system along with the respiratory tract is a potential route of COVID-19 and may have a significant impact on health policy setting regarding the prevention of COVID-19.

\section{MATERIALS AND METHODS}

\section{Data sources}

Single-cell expression matrices for the lung, oesophagus, stomach, ileum and colon were obtained from the Gene
Expression Omnibus (https://www.ncbi.nlm.nih.gov/), ${ }^{14}$ Single Cell Portal (https://singlecell.broadinstitute.org/single_cell) and Human Cell Atlas Data Portal. (https://data.humancellatlas. org). Single-cell data for the oesophagus and lung were obtained from the research published by Madissoon et al which contained six oesophageal and five lung tissue samples. ${ }^{15}$ The data of gastric mucosal samples from three non-atrophic gastritis and three chronic atrophic gastritis patients were obtained from GSE134520. ${ }^{16}$ GSE134809 ${ }^{17}$ comprises 22 ileal specimens from 11 patients with ileal Crohn's disease and only non-inflammatory samples were selected for analysis. The data from Smillie et $a l^{18}$ included 12 normal colon samples.

\section{Quality control}

Low-quality cells with fewer than 200 or greater than 5000 expressed genes were removed. We further required the percentage of unique molecular identifiers (UMIs) mapped to mitochondrial to be less than $20 \%$.

\section{Data integration, dimension reduction and cell clustering}

Different data processing methods were performed for different single-cell projects according to the downloaded data.

\section{Oesophagus and lung datasets}

Seurat ${ }^{19}$ rds data were directly downloaded from the supplementary material in Madissoon et al. ${ }^{15}$ Uniform manifold approximation and projection (UMAP) visualisation was performed to obtain clusters of cells.

\section{Stomach and ileum datasets}

a single-cell data expression matrix was processed with the $\mathrm{R}$ package Seurat (V.3.1.4). ${ }^{19}$ We first used 'NormalizeData' to normalise the single-cell gene expression data. UMI counts were normalised by the total number of UMIs per cell, multiplied by 10000 for normalisation and log-transformed. The highly variable genes (HVGs) were identified using the function 'FindVariableGenes'. We then used the 'FindIntegrationAnchors' and 'Integratedata' functions to merge multiple sample data within

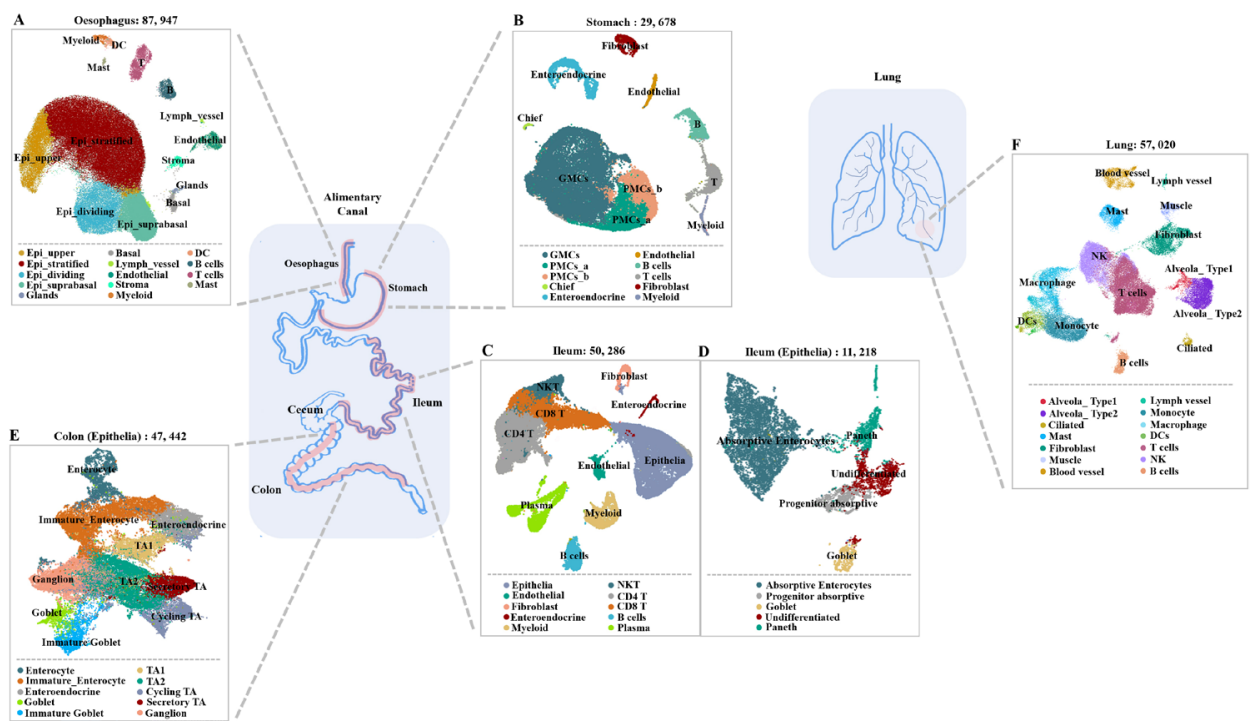

Figure 1 Single-cell atlas of digestive tract and lung tissues. (A-F) Uniform manifold approximation and projection (UMAP) plots showing the landscape of (A) oesophageal cells, (B) gastric mucosa cells, (C) ileal cells, (E) colon cells and (F) lung cells. (D) The ileal epithelial cells were further divided into finer cell subsets due to the heterogeneity within the cell population. DCs, dendritic cells; GMC, gland mucous cell; NKT, natural killer T; PMC, pit mucous cell; TA, transit amplifying. 

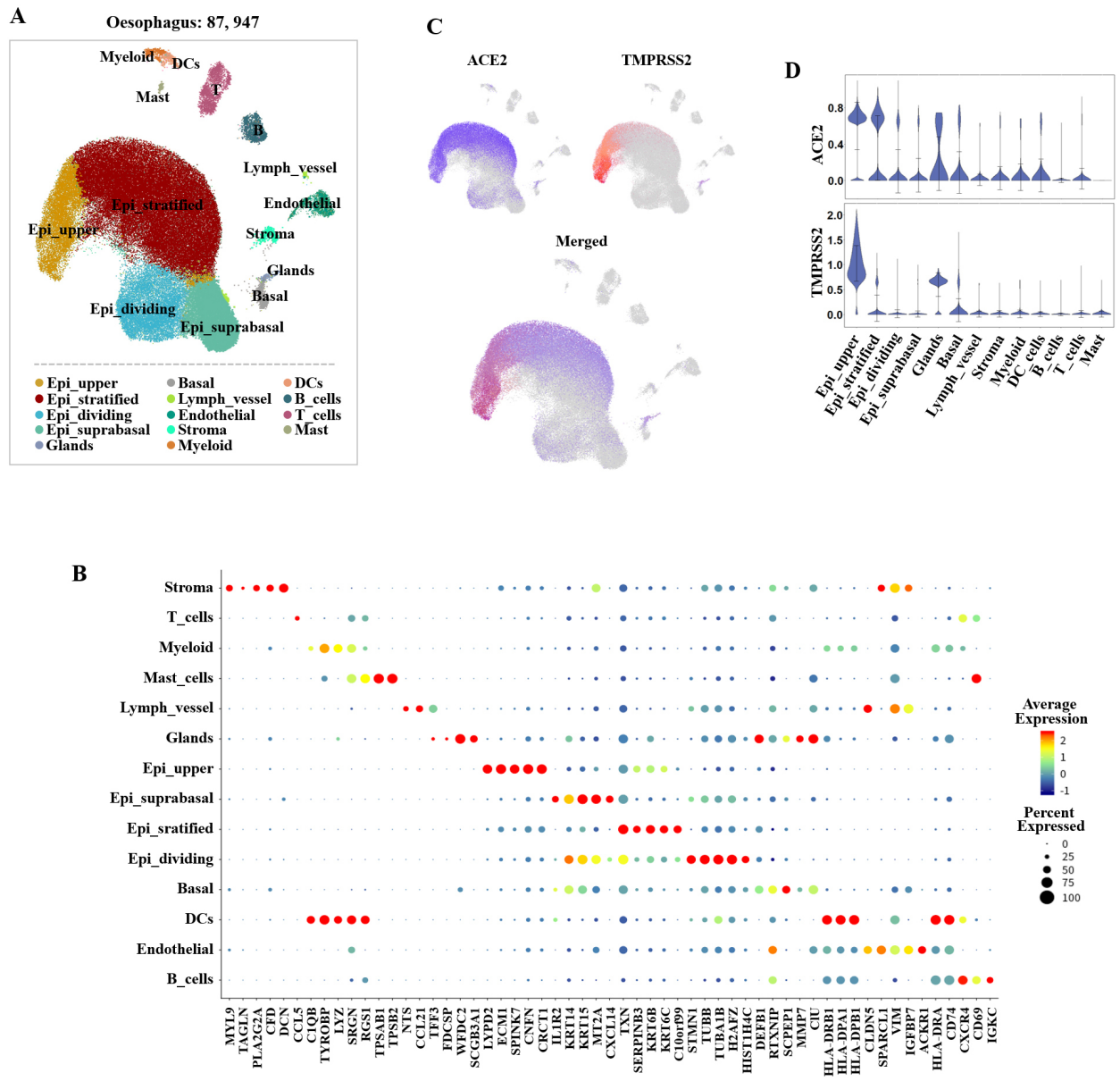

Figure 2 Single-cell analysis of oesophageal cells. (A) Uniform manifold approximation and projection (UMAP) plots showing the landscape of oesophageal cells. (B) Dot plot showing the marker genes for oesophageal cells. (C) UMAP plots showing the expression of ACE2 (blue) and transmembrane serine protease (TMPRSS2; red). The plots were merged to show the coexpression of these genes. (D) Violin plots for expression levels of ACE2 and TMPRSS2 across clusters. The expression is measured as the $\log _{2}(T P 10 K+1)$ value. DCs, dendritic cells.

each dataset. After removing unwanted sources of variation, such as cell cycle stage and mitochondrial contamination, from a single-cell dataset, we used the 'RunPCA' function to perform a principal component analysis (PCA) on the single-cell expression matrix with significant HVGs. Then, we constructed a K-nearest-neighbour graph based on the Euclidean distance in PCA space using the 'FindNeighbors' function and applied the Louvain algorithm to iteratively group cells together with the 'FindClusters' function with optimal resolution. UMAP was used for visualisation purposes.

\section{Colon dataset}

the single-cell data expression matrix was processed with the R packages LIGER ${ }^{20}$ and Seurat. ${ }^{19}$ We first normalised the data to account for differences in sequencing depth and capture efficiency among cells. Then, we used the 'selectGenes' function to identify variable genes in each dataset separately and took the union of the result. Next, integrative non-negative matrix factorisation was performed to identify shared and distinct metagenes across the datasets and the corresponding factor loading for each cell using the 'optimizeALS' function in LIGER. We selected a $\mathrm{k}$ of 15 and lambda of 5.0 to obtain a plot of expected alignment. We then identified clusters shared across datasets and aligned quantiles within each cluster and factor using the 'quantileAlignSNF' function. Next, nonlinear dimensionality reduction was performed using the 'RunUMAP' function in Seurat and the results were visualised with UMAP plots.

\section{Identification of cell types and gene expression analysis}

We annotated cell clusters based on the expression of known cell markers and the clustering information provided in the articles. Then, we used the 'RunALRA' function in Seurat to impute lost values in the scRNA-seq data. Feature plots and violin plots were generated using Seurat to show the imputed gene expression. To compare gene expression in different datasets, we used 'Quantile normalisation' in the $\mathrm{R}$ package preprocessCore ( $\mathrm{R}$ package V.1.46.0. https://github.com/bmbolstad/preprocessCore) to preprocess the data. Then, gene expression data were further denoised by adding random generation for the normal distribution with mean equal to mean and SD equal to SD

\section{External validation}

To minimise bias, external databases of Genotype-Tissue Expression (GTEx), ${ }^{21}$ and The Human Protein Atlas ${ }^{22}$ were used to detect gene and protein expression of ACE2 at the tissue level including normal lung and digestive system, such as oesophagus, stomach, small intestine and colon. 

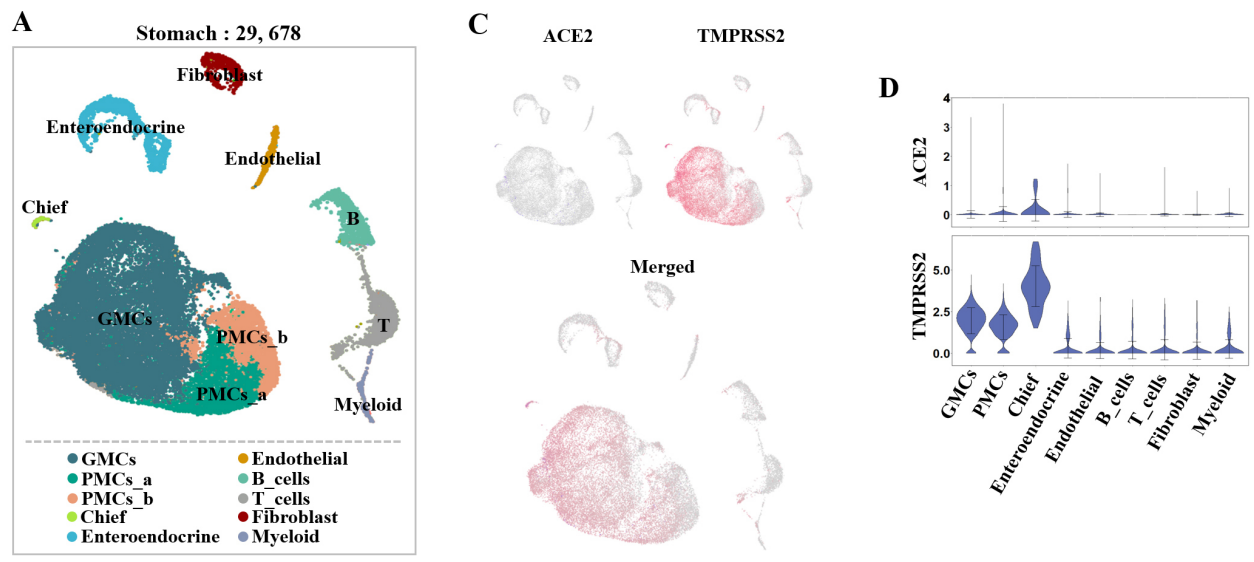

B

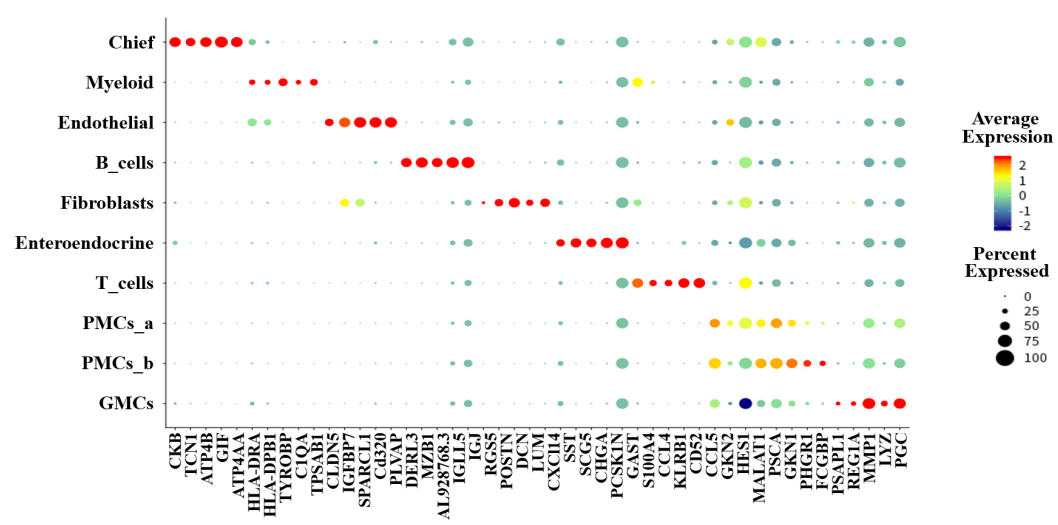

Figure 3 Single-cell analysis of gastric mucosal cells. (A) Uniform manifold approximation and projection (UMAP) plots showing the landscape of gastric mucosal cells. (B) Dot plot showing the marker genes for gastric mucosal cells. (C) UMAP plots showing the expression of ACE2 (blue) and transmembrane serine protease (TMPRSS2; red). The plots were merged to show the coexpression of these genes. (D) Violin plots for expression levels of ACE2 and TMPRSS2 across clusters. The expression is measured as the $\log _{2}(\mathrm{TP1} 10 \mathrm{~K}+1)$ value. DCs, dendritic cells; GMCs, gland mucous cells; PMCs, pit mucous cells.

\section{RESULTS}

\section{Annotation of cell types}

The gastrointestinal system is composed of the oesophagus, stomach, ileum, colon and cecum (figure 1A-E). In this study, five datasets with single-cell transcriptomes of the oesophagus, gastric mucosa, ileum and colon along with lung were analysed (online supplementary file 1 ).

In the oesophagus, 14 cell types were identified among 87947 cells. Over $90 \%$ of the cells fell into four major epithelial cell types: upper, stratified, suprabasal and dividing cells of the suprabasal layer (figure 2A). The additional cells from the basal layer of the epithelium clustered most closely with the gland duct and mucous secreting cells. Lymph vessels and endothelial cells are associated with vessel tissues. Immune cells in the oesophagus include T cells, B cells, monocytes, macrophages, dendritic cells (DCs) and mast cells. The cell type identified in oesophagus cluster was annotated by the expression of known cell-type markers (figure 2B).

A total of 29678 cells and 10 cell types were identified in the stomach after quality control with a high proportion of gastric epithelial cells, including antral basal gland mucous cells (GMCs), pit mucous cells (PMCs), chief cells and enteroendocrine cells (figure $3 \mathrm{~A}$ ). The non-epithelial cell lineages were composed of $\mathrm{T}$ cells, B cells, myeloid cells, fibroblasts and endothelial cells. The cell type identified in stomach cluster was annotated by known cell-type markers (figure 3B).
After quality controls, 50286 cells and 10 cell types were identified in the ileum. The detected cell types included epithelial, endothelial, fibroblast and enteroendocrine cells. The identified immune cell types were myeloid, $\mathrm{CD} 4^{+} \mathrm{T}, \mathrm{CD} 8^{+} \mathrm{T}$ and natural killer T cells, along with plasma and $\mathrm{B}$ cells. Among the 11218 epithelial cells (figure 4A), 5 cell types were identified, namely, absorptive enterocytes, progenitor absorptive, goblet, Paneth and undifferentiated cells by known markers (figure 4B).

All 47442 epithelial cells from the colon, including absorptive and secretory clusters, were annotated after quality controls (figure 5A). The absorptive clusters included further subclusters for transit-amplifying (TA) cells (TA 1, TA 2), immature enterocytes and enterocytes. The secretory clusters included subclusters of progenitor cells (secretory TA and immature goblet cells) and of mature cells (goblet, and enteroendocrine cells). Ganglion cells and cycling TA cells were also identified in the final UMAP. The cell types were identified by known markers (figure 5B).

After initial quality controls were performed, 57020 cells and 25 cell types were identified in the lung (figure 6A). The detected cell types included ciliated, alveolar type 1 (AT1) and -AT2 cells, along with fibroblast, muscle and endothelial cells. The identified immune cell types were T, B and NK cells, along with macrophages, monocytes and DCs. The cell types were identifiedby known markers (figure 6B). 

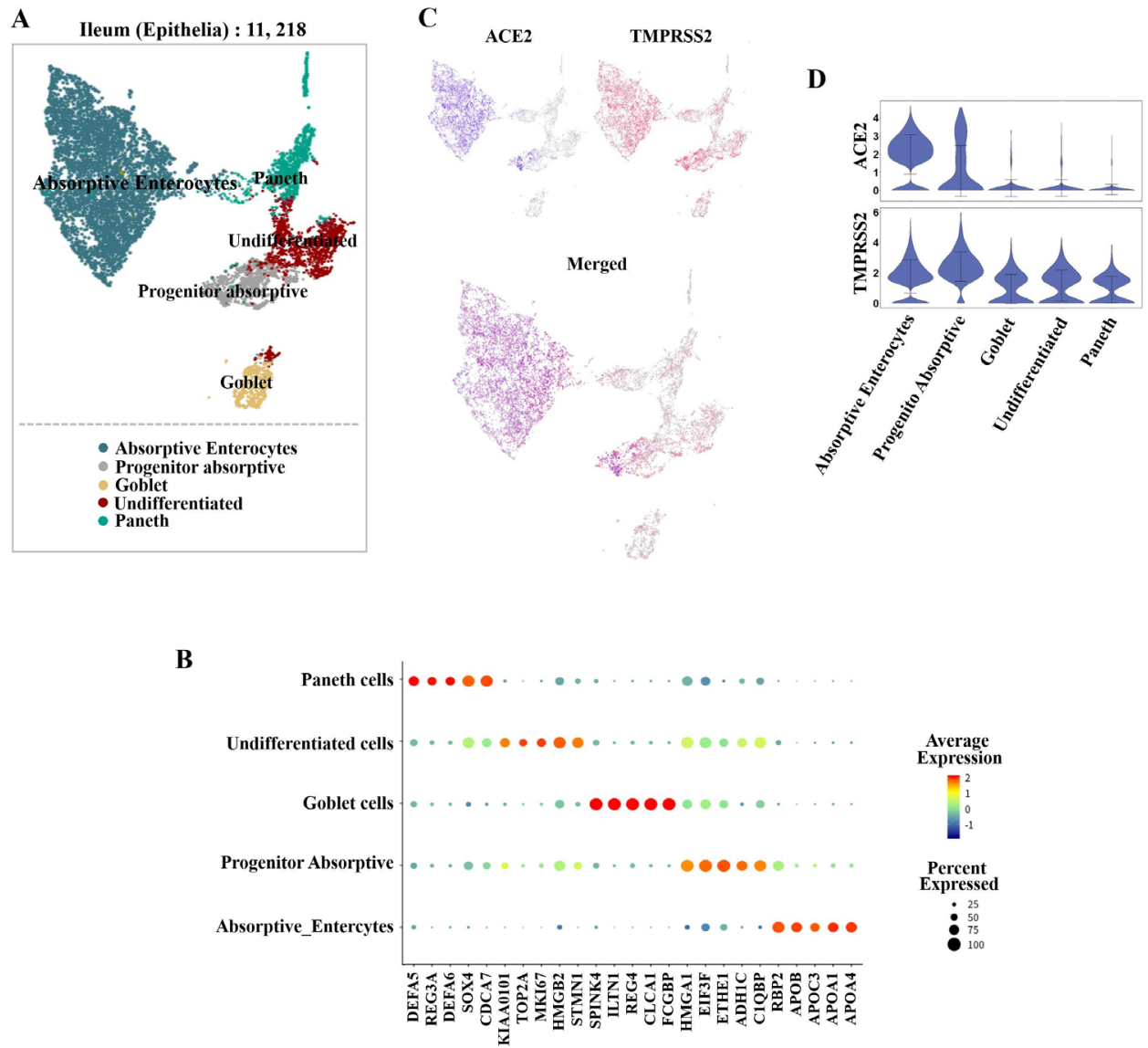

Figure 4 (Single-cell analysis of ileal epithelial cells. (A) Uniform manifold approximation and projection (UMAP) plots showing the landscape of ileal epithelial cells. (B) Dot plot showing the marker genes for ileal epithelial cells. (C) UMAP plots showing the expression of ACE2 (blue) and transmembrane serine protease (TMPRSS2; red). The plots were merged to show the coexpression of these genes. (D) Violin plots for expression levels of ACE2 and TMPRSS2 across clusters. The expression is measured as the $\log _{2}(T P 10 K+1)$ value.

\section{Cell type-specific ACE2 and TMPRSS2 expression}

To determine which cell type was the potential target cell for SARS-CoV-2 in the lung and digestive system, we explored the expression levels of ACE2 and TMPRSS2 in all cell populations. In the oesophagus, ACE2 was highly expressed in upper and stratified epithelial cells. The glands also had low ACE2 expression levels. TMPRSS2 was expressed mainly in oesophagus upper epithelial and gland cells (figure 2C). However, the stratified epithelial cells expressed TMPRSS2 at very low levels (figure 2D). With regard to the stomach, the expression of ACE2 was relatively low in all clusters (figure 3C). However, TMPRSS2 was highly expressed in GMCs, PMCs and cheif cells (figure 3C,D). In the epithelial cells of the ileum, ACE2 was highly expressed in absorptive enterocytes and expressed at lower levels in progenitor absorptive cells. TMPRSS2-expressing cells were widely distributed in all epithelial cell subclusters (figure 4C,D). As for the colon, ACE2 was mainly found in enterocytes and was expressed at lower levels in immature enterocytes. (figure 5C). TMPRSS2 also showed high expression in enterocytes and immature enterocytes, which was similar to ACE2 . Relative low expression of TMPRSS2 was also found in Goblet, Ganglion and TA cells (figure 5C,D). In the lung, we found that ACE2 was mainly expressed in AT2 cells and was also found in AT1 and fibroblast cells. TMPRSS2 was also highly expressed in AT1 and AT2 cells (figure 6C,D). Therefore, AT2 cells, which have relatively high coexpression of ACE2 and TMPRSS2, may be the main host cells for SARS-CoV-2 (figure 6C,D).
Among all the ACE2-expressing cells in the normal digestive system and lung, ACE2 was also relatively highly expressed in the ileum and colon (figure 7A). TMPRSS2 was further compared in these cells and they also showed relatively high expression in AT2, oesophagus upper and enterocytes from ileum and colon with relatively low expression in stratified epithelial cells from oesophagus (figure 7A). The RNA-seq data of the lung, oesophagus, stomach, small intestine, colon transverse and colon sigmoid were obtained from GTEx database (online supplementary file 2). ACE2 was highly expressed in small intestine and colon, with a relative low expression in lung and oesophagus (figure 7B). TMPRSS2 was highly expressed in lung, stomach, small intestine and transverse colon. However, relative low expression of TMPRSS2 was found in Sigmoid colon (figure 7B). In addition, we also collected immunohistochemical images to show the expression of ACE2 at the protein level (figure 7C). The representative pictures were derived from the lung (T-28000, patient 218), oral mucosa (T-51000, patient 48), oesophagus (T-62000, patient 3399), small intestine (T-65000, patient 493), stomach (T-63700, patient 148), duodenum (T-59000, patient 1904), colon $(\mathrm{T}-1 \times 300$, patient 3266) and rectum (T-68000, patient 1087). The results revealed that ACE2 was mainly expressed in duodenum, small intestine, colon and rectum, which was consistent with the RNA expression level (figure 7C). 

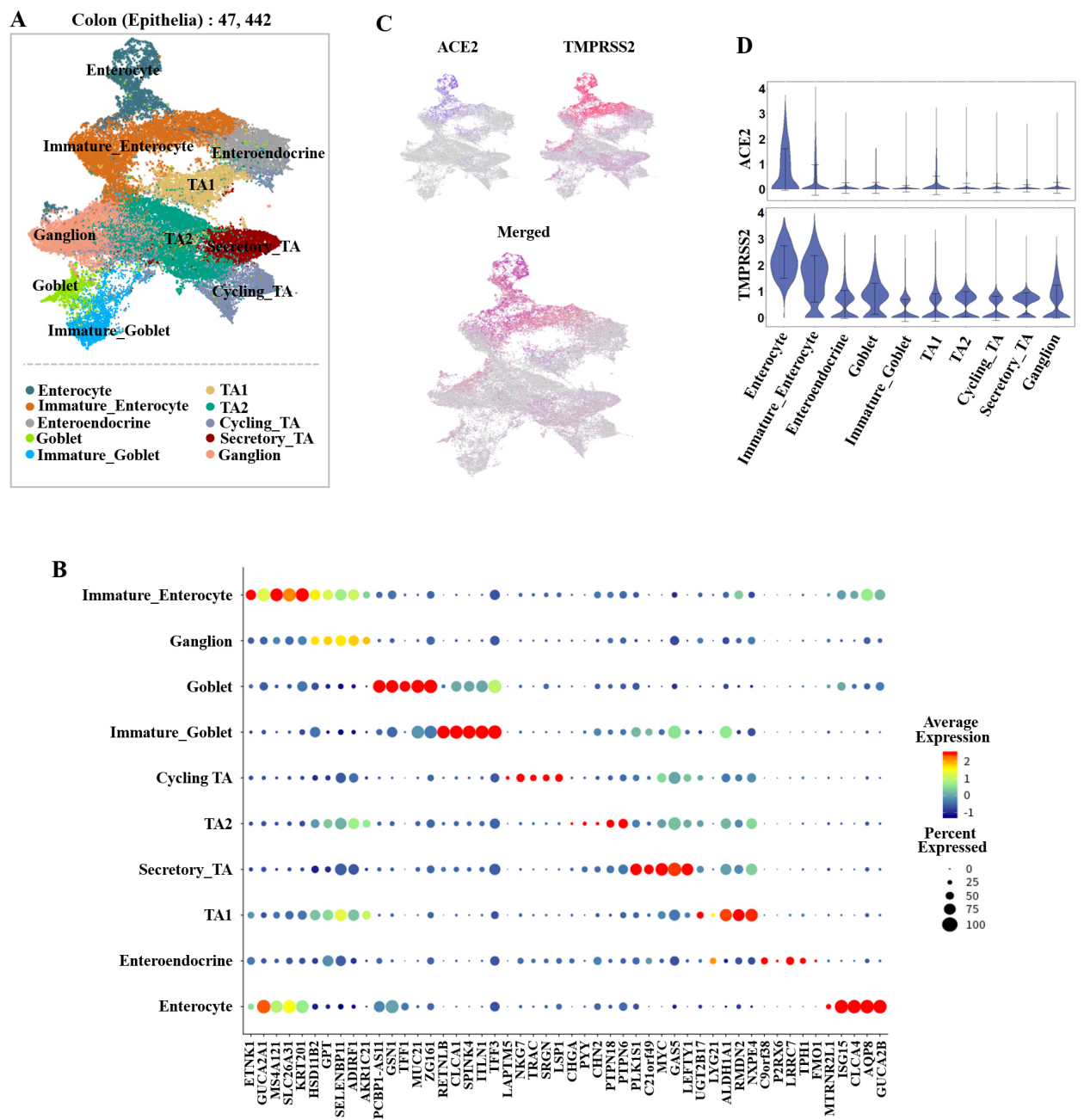

Figure 5 Single-cell analysis of colonic epithelial cells. (A) Uniform manifold approximation and projection (UMAP) plots showing the landscape of colonic epithelial cells. (B) Dot plot showing the marker genes for colonic epithelial cells. (C) UMAP plots showing the expression of ACE2 (blue) and transmembrane serine protease (TMPRSS2; red). The plots were merged to show the coexpression of these genes. (D) Violin plots for expression levels of ACE2 and TMPRSS2 across clusters. The expression is measured as the $\log _{2}(T P 10 K+1)$ value. TA, transit amplifying.

\section{DISCUSSION}

Coronaviruses are a common infection source in the upper respiratory, gastrointestinal tracts and central nervous system in humans and other mammals. ${ }^{23}$ To date, the infection routes of SARS-CoV-2 and its ability to infect the digestive system remain unclear. The virus entry process depends on the SARS-CoV receptor ACE2 and cellular serine protease TMPRSS2. It suggests that cells coexpressing ACE2 and TMPRSS2 are the most susceptible cells for infection while the cells expressing one of them remain safe. Thus far, most of the studies, expecially the single cell RNA-seq studies only focus on ACE2. ${ }^{24} 25$ Our study is the first study overlooked both of the key proteins in the virus entry process and it may provide a more comprehensive information of the potential target cell types. In addition, our study shows a novel method to identify the prime cell types of a virus by the coexpression pattern analysis of single-cell sequencing data.

In this study, we found coexpression of ACE2 and TMPRSS2 in lung AT1, AT2 cells, oesophageal upper epithelial and gland cells, and absorptive enterocytes from the ileum and colon. These findings directly show that, for the first time, ACE2 and TMPRSS2 expression is not limited to the lung and suggest that the extrapulmonary spread of SARS-CoV-2 may exist. In addition, these findings suggest that the enteric symptoms of COVID-19 may be associated with the invasion of SARS-CoC-2 into the ACE2 and TMPRSS 2 coexpressing enterocytes.

Generally, many respiratory pathogens, such as influenza, SARS-CoV and SARSr-CoV, cause enteric symptoms, as is the case for SARS-CoV-2. ${ }^{3}$ As a classic respiratory coronavirus, SARS often causes enteric symptoms along with respiratory symptoms. Moreover, transmission via stool is also a neglected risk for SARS. ${ }^{26}$ The enteric symptoms of SARS and highly pathogenic strains of influenza are associated with increased permeability to intestinal lipopolysaccharide and bacterial transmigration through the gastrointestinal wall. ${ }^{27}{ }^{28}$ However, the mechanism of SARS-CoV-2-induced enteric symptoms is still unknown.

ACE2 was found to interact with a defined receptor-binding domain of CTD1 in SARS-CoV and facilitate efficient crossspecies infection and person-to-person transmission. ${ }^{8} 29$ The 'up' and 'down' transition of CTD1 allows ACE2 binding by regulating the relationship among CTD1, CTD2, the S1-ACE2 complex and the S2 subunit. ${ }^{30}$ In human HeLa cells, expressing ACE2 from human, civet and Chinese horseshoe bats can help many kinds of SARSr-CoV, including SARS-CoV-2, enter cells, indicating the important role that ACE2 plays in cellular entry. ${ }^{9}$ 31-33 Structural analysis of the SARS-CoV-2 S protein 

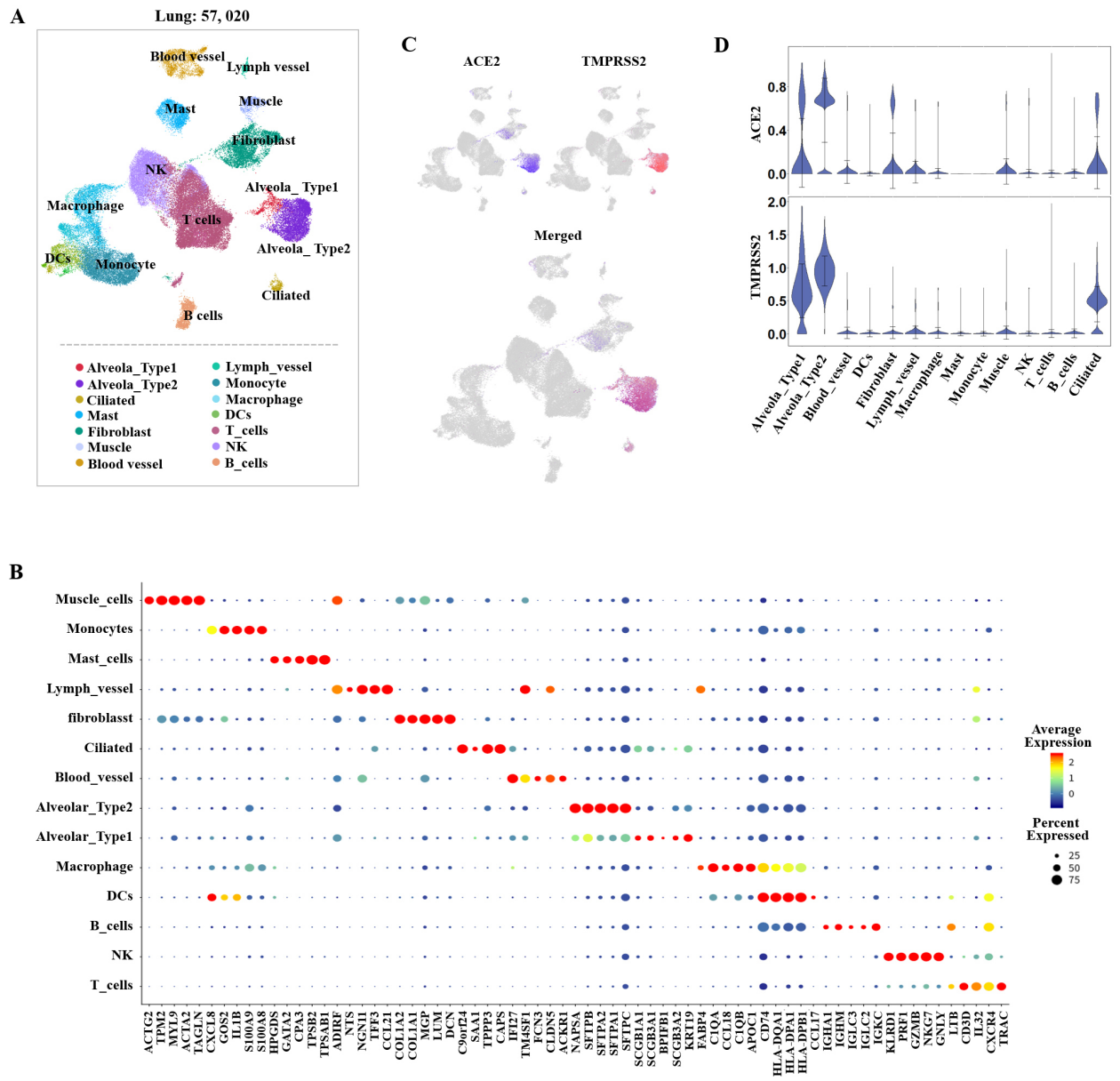

Figure 6 Single-cell analysis of lung cells. (A) Uniform manifold approximation and projection (UMAP) plots showing the landscape of lung cells. (B) Dot plot showing the marker genes for lung cells. (C) UMAP plots showing the expression of ACE2 and transmembrane serine protease (TMPRSS2). The plots were merged to show the coexpression of these genes. (D) Violin plots for expression levels of ACE2 and TMPRSS2 across clusters. The expression is measured as the $\log _{2}(T P 10 K+1)$ value. DC, dendritic cell; NK, natural killer.

that it binds ACE2 with higher affinity than does SARS CoV S protein. ${ }^{34}$

The TMPRSS2 can cleave SARS-S protein and render host cell entry independent of the endosomal pathway using cathepsin $\mathrm{B} / \mathrm{L} .{ }^{35}$ Different from cathepsin $\mathrm{B} / \mathrm{L}$, they can also promote viral spread in the host and cleave ACE2 to augment about 30 -fold viral infectivity. ${ }^{36}{ }^{37}$ In addition, the key sequence of SARS-CoV-2 spike protein cleavage has higher furin score (0.688) than thecorresponding sequence in SARS-CoV (0.139), which increases its infectivity. ${ }^{38}$ Serine protease inhibitor could also block SARS-CoV-2 infection of lung cells. ${ }^{13}$

By analysing the coexpression of ACE2 of TMPRSS2 in the normal human gastrointestinal system and lung, we identified AT2 cells the most susceptible cells in the lung due to its high expression of ACE2 and TMPRRS2. AT1cells could also be the host cells of infection, which have relatively lower expression than AT2 cells. These results were consistent with the findings of a previous study. ${ }^{39}$ In lung alveoli, AT1 epithelial cells are responsible for gas exchange and AT2 cells are in charge of surfactant biosynthesis and self-renewal. ${ }^{40}$ In SARS-CoV infection, AT2 is the major infected cell type, as assessed by viral antigen and secretory vesicle detection. Its expression in AT2 cells is variable in different donors, which may be associated with susceptibility and seriousness differences. ${ }^{39}$ Thus, we hypothesise that AT2 cells might be the key SARS-CoV-2-invaded cells in the lung and the number of AT2 cells might be associated with the severity of respiratory symptoms.

Besides the lung, coexpression of ACE2 and TMPRSS2 was found in oesophageal upper and gland cells and absorptive enterocytes from the ileum and colon. Histologically, both oesophageal and respiratory system organs, such as the trachea and lung, originate from the anterior portion of the intermediate foregut. ${ }^{41}$ After separating from the neighbouring respiratory system, the oesophagus undergoes subsequent morphogenesis from a simple columnar-to-stratified squamous epithelium. ${ }^{42}$ The upper epithelium can be nourished by submucosal glands and sustain the passage of abrasive raw food. In Barrett's oesophagus, acid reflux-induced oesophagitis and the multilayered epithelium are associated with upper epithelial cells. ${ }^{43}$ In the digestive system, in addition to being expressed in oesophageal upper epithelial and gland cells, coexpression of ACE2 and TMPRSS2 was also found in the absorptive enterocytes from the ileum and colon, the most vulnerable intestinal epithelial cells. During microbial infections, intestinal epithelial cells function as a barrier and help coordinate immune responses. ${ }^{44}$ The absorptive enterocytes can be infected by coronavirus, rotavirus and noroviruses, resulting in diarrhoea by absorptive enterocytes destruction, malabsorption, unbalanced intestinal secretion and enteric nervous system activation. ${ }^{45-47}$ Although most virus would be dead in the strong acid environment in the stomach, there is still a possibility that 

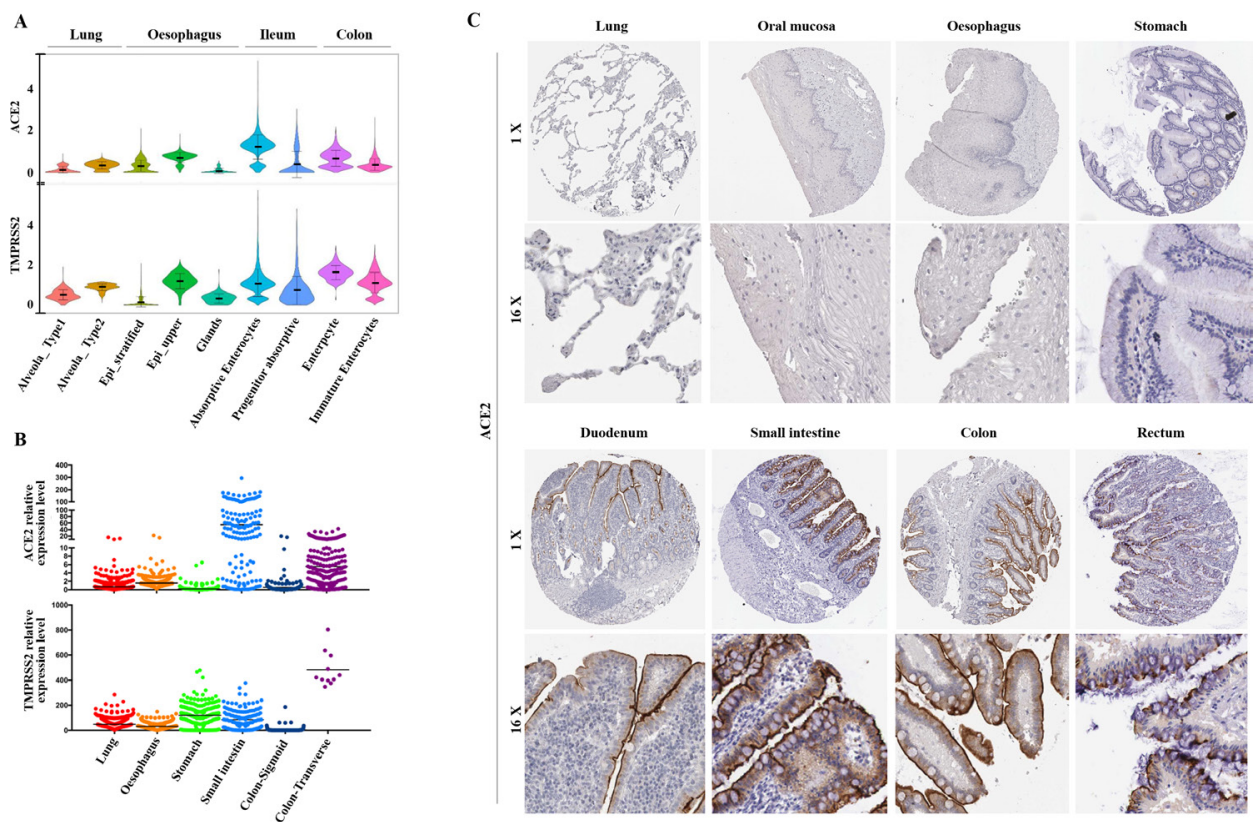

Figure 7 Expression of ACE2 and transmembrane serine protease (TMPRSS2) at RNA and protein levels in different tissues. (A) Violin plots for ACE2 and TMPRSS2 expression across two lung clusters and seven digestive tract clusters. The gene expression matrix was normalised and denoised to remove unwanted technical variability across the four datasets. (B) External validation of ACE2 and TMPRSS2 at RNA level in different tissues. The expression is measured as the pTPM value in the RNA-seq data from the Genotype-Tissue Expression database. (C) Representative immunohistochemical images of ACE2 in different tissues from the HumanProtein Atlas database.

the saliva and secretions could carry the virus into the digestive tract where viral replication may be sustained in these susceptible cells. Thus, the enteric symptom of diarrhoea might be associated with the infected ACE2-expressing and TMPRSS2expressing enterocytes. This could also help explain the fact that $10 \%$ of patients presented with diarrhoea and nausea 1 or 2 days before the development of fever and respiratory symptoms. ${ }^{6}$

Moreover, due to the high expression of ACE2 and TMPRSS2 in oesophageal upper cells and absorptive enterocytes from the ileum and colon, we propose that the digestive system could be invaded by SARS-CoV-2 and might serve as a route of infection. This supposition was supported by the first case of SARS-CoV-2 in the USA, whose stool specimen obtained on illness day 7 was detected SARS-CoV-2 RNA. ${ }^{48}$ SARS-CoV-2 was also isolated from a stool specimen of a confirmed case in China. ${ }^{49}$ additionallyThe evidence that live virus in stool specimens further supports our hypothesis.

\section{CONCLUSION}

This single-cell transcriptomic study provides the evidence of the potential route of SARS-CoV-2 in the digestive system along with the respiratory tract. It may have a significant impact to health policy setting regarding the prevention of SARS-CoV-2 infection. In addition, our study provides a novel method to guide identification of prime cell types of a virus by thecoexpression pattern analysis of single-cell sequencing data.

\section{Author affiliations}

'Department of Rheumatology and Immunology, Changzheng Hospital, Second Military Medical University, Shanghai, China

${ }^{2}$ Department of Orthopaedic Oncology, Shanghai Changzheng Hospital, Second Military Medical University, Shanghai, China

${ }^{3}$ Oiu-Jiang Bioinformatics Institute, Shanghai, China

${ }^{4}$ Department of Urology, The Third Affiliated Hospital of Second Military Medical University, Shanghai, China

${ }^{5}$ Department of Neurosurgery, Changhai Hospital, Second Military Medical University, Shanghai, China
${ }^{6}$ Translational Genomics Group, Institute of Health and Biomedical Innovation, Queensland University of Technology, Translational Research Institute, Brisbane, Queensland, Australia

${ }^{7}$ Institute for Glycomics, Griffith University, Southport, QLD, Australia

${ }^{8}$ Division of Spine, Department of Orthopedics, Tongji Hospital affiliated to Tongji University School of Medicine, Shanghai, China

${ }^{9}$ Tongji University Cancer Center, School of Medicine, Tongji University, Shanghai, China

${ }^{10}$ Peking-Tsinghua Center for Life Sciences, Tsinghua University, Beijing, China ${ }^{11}$ Beijing Tsinghua Changgeng Hospital, School of Clinical Medicine, Tsinghua University, Beijing, China

Contributors $\mathrm{WZ}$ and $\mathrm{HZ}$ : study design. $\mathrm{HZ}, \mathrm{WZ}, \mathrm{HG}$ and $\mathrm{DX}$ : data analysis. ZL, ZK, HG and DX: data collection and generation. JZ, JW, ZL, XC and JX: data interpretation. TM, ZK and HX: manuscript drafting. JZ, TM, WZ, JL and HX: overall supervising and organising the study.

Funding HX was supported by the National Natural Science Foundation of China (Grant 31821003) and the China Ministry of Science and Technology (Grant 2018AAA0100300).

Competing interests None declared.

Patient and public involvement Patients and/or the public were not involved in the design, or conduct, or reporting, or dissemination plans of this research.

\section{Patient consent for publication Not required.}

Ethics approval There is no direct involvement of human subjects in this project. All the data use existing deidentified biological samples and data from prior studies. Therefore, ethical oversight and patient consent were not handled in this study.

Provenance and peer review Not commissioned; externally peer reviewed.

Data availability statement Single cell data can be obtained in Human Cell Atalas accession code PRJEB31843 (https://data.humancellatlas.org/ explore/projects/c4077b3c-5c98-4d26-a614-246d12c2e5d7), Gene Expression Omnibus(GSE134520 and GSE134809) and Single Cell Portal accession code SCP259 ( https://singlecell.broadinstitute.org/single_cell/study/SCP259/intraand-inter-cellular-rewiring-of-the-human-colon-during-ulcerative-colitis). All data relevant to the study are descripted in detail in the article or uploaded as supplementary information.

Open access This is an open access article distributed in accordance with the Creative Commons Attribution Non Commercial (CC BY-NC 4.0) license, which permits others to distribute, remix, adapt, build upon this work non-commercially, and license their derivative works on different terms, provided the original work is 
properly cited, appropriate credit is given, any changes made indicated, and the use is non-commercial. See: http://creativecommons.org/licenses/by-nc/4.0/.

\section{ORCID iD}

Tong Meng http://orcid.org/0000-0001-6403-1357

\section{REFERENCES}

1 The Lancet. Emerging understandings of 2019-nCoV. Lancet 2020;395:311.

2 Zhu N, Zhang D, Wang W, et al. A novel coronavirus from patients with pneumonia in China, 2019. N Engl J Med 2020;382:727-33.

3 Chan JF-W, Yuan S, Kok K-H, et al. A familial cluster of pneumonia associated with the 2019 novel coronavirus indicating person-to-person transmission: a study of a family cluster. Lancet 2020;395:514-23.

4 Huang C, Wang Y, Li X, et al. Clinical features of patients infected with 2019 novel coronavirus in Wuhan, China. Lancet 2020;395:497-506.

5 Chen N, Zhou M, Dong X, et al. Epidemiological and clinical characteristics of 99 cases of 2019 novel coronavirus pneumonia in Wuhan, China: a descriptive study. Lancet 2020;395:507-13.

6 Wang D, Hu B, Hu C, et al. Clinical characteristics of 138 hospitalized patients with 2019 novel coronavirus-infected pneumonia in Wuhan, China. JAMA 2020. doi:10.1001/jama.2020.1585. [Epub ahead of print: 07 Feb 2020].

7 Li F. Structure, function, and evolution of coronavirus spike proteins. Annu Rev Virol 2016;3:237-61.

8 Gui M, Song W, Zhou H, et al. Cryo-Electron microscopy structures of the SARS-CoV spike glycoprotein reveal a prerequisite conformational state for receptor binding. Cell Res 2017:27:119-29.

9 Zhou P, Yang X-L, Wang X-G, et al. A pneumonia outbreak associated with a new coronavirus of probable bat origin. Nature 2020;579:270-3.

$10 \mathrm{Xu} \mathrm{X}$, Chen P, Wang J, et al. Evolution of the novel coronavirus from the ongoing Wuhan outbreak and modeling of its spike protein for risk of human transmission. $\mathrm{SC}$ China Life Sci 2020;63:457-60.

11 Wan Y, Shang J, Graham R, et al. Receptor recognition by the novel coronavirus from Wuhan: an analysis based on decade-long structural studies of SARS coronavirus. J Virol 2020;94. doi:10.1128/JVI.00127-20

12 Gallagher TM, Buchmeier MJ. Coronavirus spike proteins in viral entry and pathogenesis. Virology 2001;279:371-4.

13 Hoffmann M, Kleine-Weber $H$, Schroeder $\mathrm{S}$, et al. SARS-CoV-2 cell entry depends on ACE2 and TMPRSS2 and is blocked by a clinically proven protease inhibitor. Cell 2020. doi:10.1016/j.cell.2020.02.052

14 Edgar R, Domrachev M, Lash AE. Gene expression Omnibus: NCBI gene expression and hybridization array data Repository. Nucleic Acids Res 2002;30:207-10.

15 Madissoon E, Wilbrey-Clark A, Miragaia RJ, et al. scRNA-Seq assessment of the human lung, spleen, and esophagus tissue stability after cold preservation. Genome Biol 2019;21:1.

16 Zhang P, Yang M, Zhang Y, et al. Dissecting the single-cell transcriptome network underlying gastric premalignant lesions and early gastric cancer. Cell Rep 2019;27:1934-47.

17 Martin JC, Chang C, Boschetti G, et al. Single-Cell analysis of Crohn's disease lesions identifies a pathogenic cellular module associated with resistance to anti-TNF therapy. Cell 2019;178:1493-508.

18 Smillie CS, Biton M, Ordovas-Montanes J, et al. Intra- and Inter-cellular rewiring of the human colon during ulcerative colitis. Cell 2019;178:714-30.

19 Stuart T, Butler A, Hoffman P, et al. Comprehensive integration of single-cell data. Cell 2019;177:1888-902.

20 Welch JD, Kozareva V, Ferreira A, et al. Single-Cell multi-omic integration compares and contrasts features of brain cell identity. Cell 2019;177:1873-87.

21 GTEx Consortium. Human genomics. The Genotype-Tissue expression (GTEx) pilot analysis: multitissue gene regulation in humans. Science 2015;348:648-60.

22 Uhlen M, Fagerberg L, Hallstrom BM, et al. Tissue-Based map of the human proteome. Science 2015:347:1260419.

23 Perlman S, Netland J. Coronaviruses post-SARS: update on replication and pathogenesis. Nat Rev Microbiol 2009;7:439-50.
24 Zou X, Chen K, Zou J, et al. Single-Cell RNA-seq data analysis on the receptor ACE2 expression reveals the potential risk of different human organs vulnerable to 2019 nCoV infection. Front Med 2020. doi:10.1007/s11684-020-0754-0

25 Chai X, Hu L, Zhang Y, et al. Specific ACE2 expression in cholangiocytes may cause liver damage after 2019-nCoV infection. BioRxiv2020.

26 Peiris JSM, Chu CM, Cheng VCC, et al. Clinical progression and viral load in a community outbreak of coronavirus-associated SARS pneumonia: a prospective study. Lancet 2003;361:1767-72.

27 Powers JH, Bacci ED, Guerrero ML, et al. Reliability, validity, and responsiveness of influenza patient-reported outcome (FLU-PROC) scores in Influenza-Positive patients. Value Health 2018;21:210-8.

28 To KF, Tong JHM, Chan PKS, et al. Tissue and cellular tropism of the coronavirus associated with severe acute respiratory syndrome: an in-situ hybridization study of fatal cases. J Pathol 2004;202:157-63.

29 Li F, Li W, Farzan M. Structure of SARS coronavirus spike receptor-binding domain complexed with receptor. Science 2005;309:1864-8.

30 Song W, Gui M, Wang X, et al. Cryo-Em structure of the SARS coronavirus spike glycoprotein in complex with its host cell receptor ACE2. PLOS Pathog 2018:14:e1007236.

31 Yang $X-L$, Hu B, Wang B, et al. Isolation and characterization of a novel bat coronavirus closely related to the direct progenitor of severe acute respiratory syndrome coronavirus. J Virol 2015;90:3253-6.

32 Ge X-Y, Li J-L, Yang X-L, et al. Isolation and characterization of a bat SARS-like coronavirus that uses the ACE2 receptor. Nature 2013;503:535-8.

33 Hu B, Zeng L-P, Yang X-L, et al. Discovery of a rich gene pool of bat SARS-related coronaviruses provides new insights into the origin of SARS coronavirus. PLoS Pathog 2017; 13:e1006698.

34 Wrapp D, Wang N, Corbett KS, et al. Cryo-Em structure of the 2019-nCoV spike in the prefusion conformation. Science 2020;367:1260-3.

35 Shirato K, Kawase M, Matsuyama S. Wild-Type human coronaviruses prefer cellsurface TMPRSS2 to endosomal cathepsins for cell entry. Virology 2018;517:9-15.

36 Heurich A, Hofmann-Winkler H, Gierer S, et al. Tmprss2 and ADAM17 cleave ACE2 differentially and only proteolysis by TMPRSS2 augments entry driven by the severe acute respiratory syndrome coronavirus spike protein. J Virol 2014;88:1293-307.

37 Zhou Y, Vedantham P, Lu K, et al. Protease inhibitors targeting coronavirus and filovirus entry. Antiviral Res 2015;116:76-84.

38 Meng T, $\mathrm{CaO} \mathrm{H}$, Zhang H, et al. The insert sequence in SARS-CoV-2 enhances spike protein cleavage by TMPRSS. bioRxiv2020.

39 Qian Z, Travanty EA, Oko L, et al. Innate immune response of human alveolar type II cells infected with severe acute respiratory syndrome-coronavirus. Am J Respir Cell Mol Biol 2013;48:742-8.

40 Nabhan AN, Brownfield DG, Harbury PB, et al. Single-Cell Wnt signaling niches maintain stemness of alveolar type 2 cells. Science 2018;359:1118-23.

41 Que J, Okubo T, Goldenring JR, et al. Multiple dose-dependent roles for Sox2 in the patterning and differentiation of anterior foregut endoderm. Development 2007;134:2521-31.

42 Zhang $Y$, Jiang $M$, Kim E, et al. Development and stem cells of the esophagus. Semin Cell Dev Biol 2017;66:25-35.

43 Jiang M, Li H, Zhang Y, et al. Transitional basal cells at the squamous-columnar junction generate Barrett's oesophagus. Nature 2017;550:529-33.

44 Haber AL, Biton M, Rogel N, et al. A single-cell survey of the small intestinal epithelium. Nature 2017;551:333-9.

45 Crawford SE, Ramani S, Tate JE, et al. Rotavirus infection. Nat Rev Dis Primers 2017;3:17083

46 Ettayebi K, Crawford SE, Murakami K, et al. Replication of human noroviruses in stem cell-derived human enteroids. Science 2016:353:1387-93.

47 Desmarets LMB, Theuns S, Roukaerts IDM, et al. Role of sialic acids in feline enteric coronavirus infections. J Gen Virol 2014;95:1911-8.

48 Holshue ML, DeBolt C, Lindquist S, et al. First case of 2019 novel coronavirus in the United States. N Engl J Med 2020;382:929-36.

49 Guan W-jie, Ni Z-yi, Hu Y, et al. Clinical characteristics of coronavirus disease 2019 in China. New England Journal of Medicine 2020 